International Journal of Engineering \& Technology, 7 (3.13) (2018) 95-98
International Journal of Engineering \& Technology
SPC
Website: www.sciencepubco.com/index.php/IJET
Research paper

\title{
Miniaturized Band Pass Filter in Substrate Integrated WaveguideTechnology
}

\author{
Ananya Parameswaran ${ }^{1 *}$, Athira $\mathrm{P}^{2}$, S. Raghavan ${ }^{3}$ \\ ${ }^{l}$ Department of ECE NIT Trichy \\ ${ }^{2}$ Department of ECE, NIT Trichy \\ ${ }^{3}$ Department of ECE NIT Trichy \\ *Corresponding author: anus.puthen@gmail.com
}

\begin{abstract}
Miniaturization is an important criteria in the selection of devices for next generation communication systems. A novel miniaturization technique for an inductive post filter is investigated in this paper. Miniaturization is not so popular in inductive post Substrate Integrated Waveguide (SIW) filter and so considerable amount of research is to be done in this domain. A band pass filter in SIW technology using inductive posts is realized and further analyses are carried out. The insertion loss in the pass band is found to be $0.5 \mathrm{~dB}$ and the return loss is $22 \mathrm{~dB}$. In this paper, we investigate the use of slow wave technology for miniaturization. Unlike the conventional SIW, the slow wave SIW topology requires a double layer substrate with internal metallized vias introduced in the bottom layer connected to the bottom conductive plane. The number of rows of internal metallized vias was chosen based on a parametric study. The proposed miniaturization technique shows that the SIW filter is $21.6 \%$ and $34.6 \%$ miniaturized in size and area respectively. The response of the filter covers Ka band and hence is suitable for satellite communication application. A quality factor of 506 is achieved for the miniaturized filter.
\end{abstract}

Keywords: Slow wave; internal blind vias; band pass filter; SIW

\section{Introduction}

Recent decades have witnessed evolution of various technologies for the development of microwave and millimeter wave components. With rapid development of such systems, performance requirements of passive band pass filters (as an essential part in these systems) are steadily increasing. SIW is a favorable choice to design band pass filters in microwave and millimeter wave frequencies. With its compact nature and low loss characteristics, SIW has attracted scientists and researchers working in the microwave and millimeter wave domains. SIW components inherit almost all advantages of conventional wave-guides such as complete shielding, low loss, high quality-factor and high powerhandling capability overcoming their possible disadvantages such as large size and high fabrication cost. Similar to micro-strip and coplanar lines, SIW components are compact, light, easy to fabricate, flexible, and cost effective. SIW band pass filters could be designed using Complementary split ring resonators (CSRR). Here the high pass nature of SIW together with the band stop nature of CSRR is considered for the band pass response [1-4]. Band pass filter with SIW could also be realized using Defected Ground Structures (DGS). The basic concept is similar to obtaining band pass response using SIW and CSRR[5 -6]. Band pass filter with a combination of CSRR and DGS in SIW is also proposed [2]. Introducing inductive posts and irises in SIW can also result in a band pass filter [7-13,16,19]. The next generation telecommunication systems have a great demand for compact devices which made miniaturization an important criteria. A few techniques for miniaturization are illustrated in [1-6,16,23]. In [1,3], compact size was achieved by incorporating the CSRR, resulting in an increased electrical length, thereby giving a band pass filter in the lower $\mathrm{GHz}$ range. In $[2,4]$, miniaturization was made possible by the presence of DGS in the structure. Another approach for miniaturization in SIW components is by exploiting the concept of half mode as studied in $[5,6,16]$ where a size reduction of atleast $50 \%$ is possible. In $[1-6,16]$, cavity filters were dealt with where the amount of losses will be less $<2 \mathrm{~dB}$. Dealing with open ended structures at higher frequencies $>30 \mathrm{GHz}$ is little challenging as more losses account into. In this paper, the authors propose a technique to achieve size reduction for SIW inductive post band pass filter (an open ended structure) by using a phenomenon of separation of electric and magnetic fields. In [23], capacitance and inductance are due to proximity coupling in terms of fringe fields whereas in the proposed work they are due to the physical separation between electric and magnetic fields . In this work, we design a SIW band pass filter using inductive posts and propose a new technique for filter miniaturization known as slow wave phenomenon. The concept of slow wave, not used before for miniaturization of inductive post band pass filter, shows that it is possible to achieve size reduction, while maintaining almost the same filter characteristics. The paper is organized as follows. Section 2 deals with basics of SIW structure followed by Section 3 illustrating basic SIW filter design. Section 4 describes slow-wave effect.It also contains performance comparison between basic SIW filter and slow wave SIW filter performances is shown. Full wave simulation based on a Finite Difference Time Domain (FDTD) package is used for analyzing the structure. Finally, the work is concluded in Section 5. 


\section{SIW Structure}

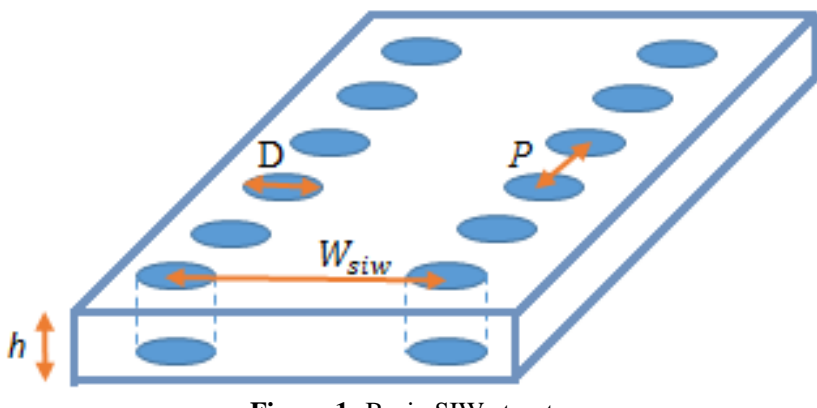

Figure 1: Basic SIW structure

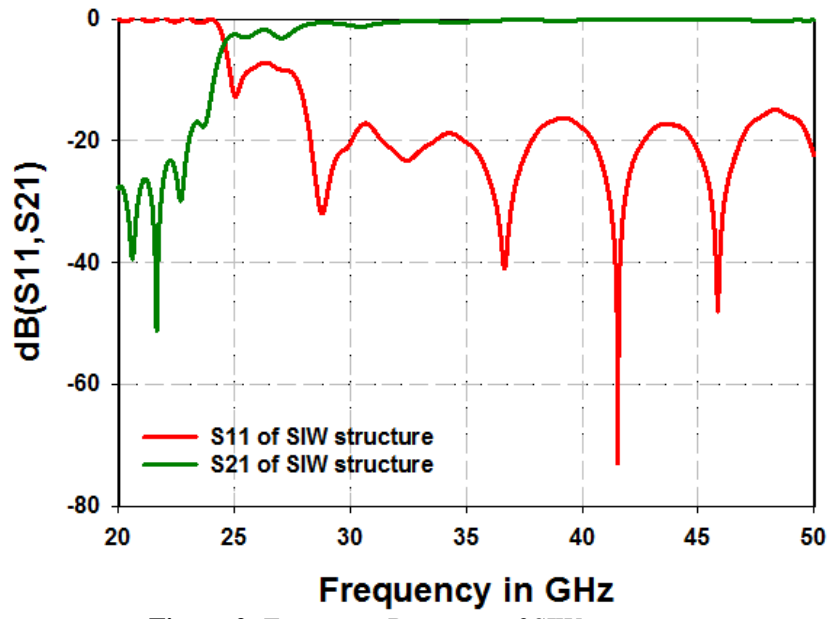

Figure 2: Frequency Response of SIW structure

Basic SIW structure consists of a substrate of height $h$, with top and bottom metallic conductive layers and lateral vias as shown in Figure 1. Lateral vias act as electric wall for the wave guide structure $[15,18]$. Like any wave guiding structure, it acts as a high pass filter with a lower cut-off frequency, as shown in Figure 2. The SIW considered in this work is designed for a cut off frequency of $25 \mathrm{GHz}$ as apparent from its S21 component of frequency response. Rogers RO5880 substrate with a relative permittivity of 2.2 is used for the filter analysis. Other design parameters considered are, height of the substrate, $\mathrm{h}=0.254 \mathrm{~mm}$, diameter of via, $\mathrm{D}$ $=0.25 \mathrm{~mm}$, distance between vias, pitch, $\mathrm{P}=0.4 \mathrm{~mm}$ and width of SIW, Wsiw $=4 \mathrm{~mm}$.

\section{Siw Band Pass Filter Design}

The basic SIW filter discussed above is considered for designing a band pass filter. The filter layout is as shown in Figure 3. Tapered sections are used for proper impedance matching. The tapered sections also help in mode conversion between micro-strip feed and SIW. The mode present in the former is Transverse Magnetic (TM) and that present in latter is Transverse Electric (TE). Introducing inductive posts into the basic SIW filter converts the high pass into a band pass response. The equivalent circuit of the inductive post is as shown in Figure 4. The effect of introducing inductive posts in basic wave-guide could be referred in $[8,9,12,22]$. The inductive posts together with basic SIW wave-guide gives a band pass response [22]. The diameters of the inductive posts and distance between adjacent posts were optimized in order to achieve a perfect pass band. The frequency response of the basic SIW filter is as shown in Figure 5. The insertion loss in pass band is $0.5 \mathrm{~dB}$, return loss is $22 \mathrm{~dB}$ and $3 \mathrm{~dB}$ bandwidth is $2.3 \mathrm{GHz}$.
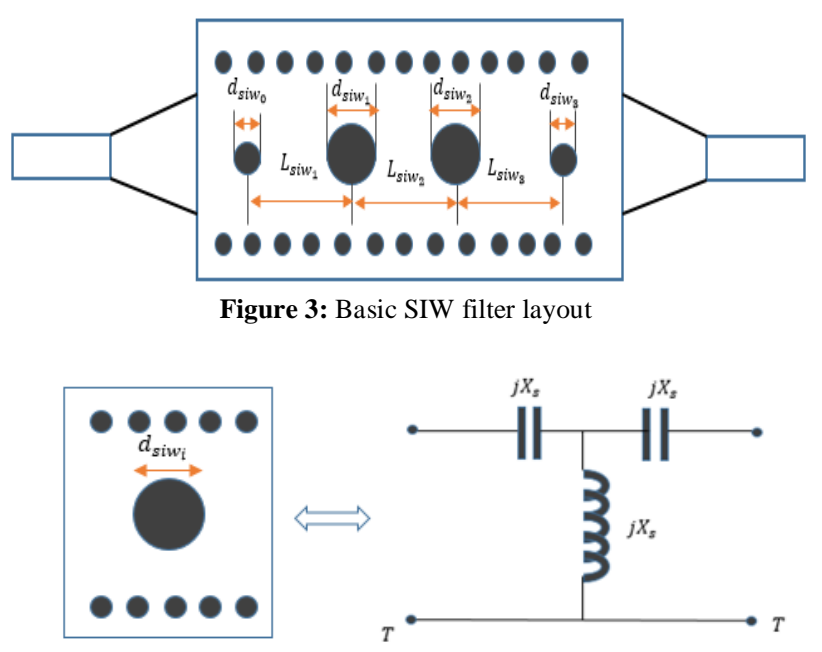

Figure 4: Equivalent circuit of an inductive post in SIW filter

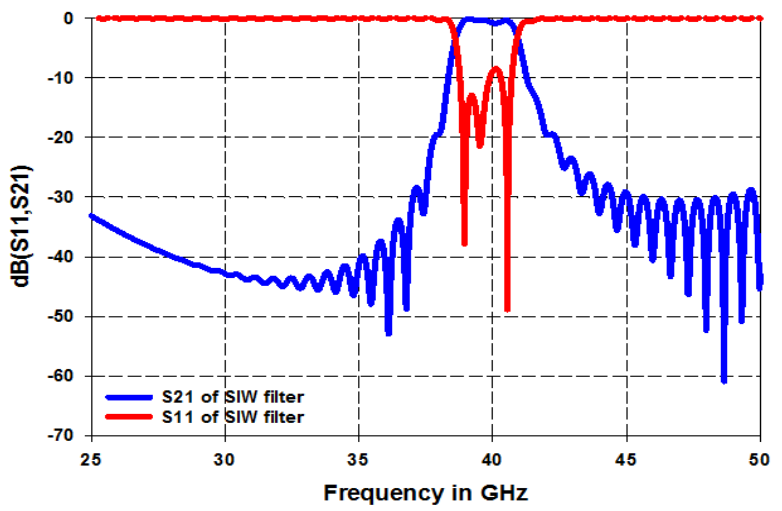

Figure 5: Frequency response of SIW filter

\section{Slow Wave Siw Filter Design}

Introducing blind vias in the pre-designed filter can reduce the filter size thus resulting in a slow wave SIW band pass filter. The slow wave concept is based on physical separation of electric and magnetic fields [15]. A lateral view of slowwave SIW filter is shown in Figure 6. The electric field is present in the top substrate of height $\mathrm{h} 1$ and the magnetic field is present all around the internal blind vias. The internal vias in the substrate 2 act as reactive loading. Substrate 1 is an isolating layer preventing short circuiting between the circuits to be hosted by the slow wave structure [20]. Circuits and slow wave structure together are essentially planar in nature and can be fabricated by the standard Printed Circuit Board (PCB) technology.

The number of rows of internal vias to be used was based on a parametric study. Analysis was done using five rows and three rows of internal vias. The value of S21 with five rows of internal vias was below $10 \mathrm{~dB}$ whereas it was around $2.5 \mathrm{~dB}$ with three rows of internal blind vias. This means that the amount of losses is more with five rows of internal vias in comparison with three rows of internal blind vias. So, further analysis was carried out using three rows of internal blind vias. Introducing blind internal vias beneath the feeds was done by investigating the effect on a simple 


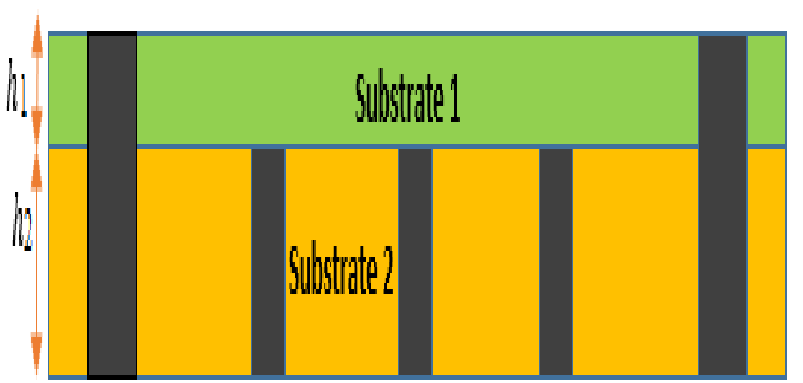

Figure 6: Lateral view of slow wave SIW structure

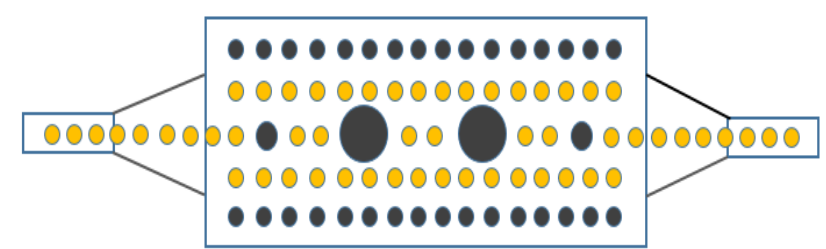

Figure 7: Slow wave SIW filter structure

micro-strip line since input is fed to the filter using a micro-strip line. Analysis showed that the internal vias need to be given just beneath the feed thereby reducing fabrication complexity and cost. Hence single row of internal vias is used in the feed sections. The final slow wave SIW band pass filter layout is shown in Figure 7.

The slow wave phenomenon leads to an increase in the effective inductance and capacitance associated with the structure as shown in Figure 8. The increase in inductance and capacitance also makes the structure para-electric and paramagnetic in nature by increasing the effective permittivity $\varepsilon$ and effective permeability $\mu$ [20]. This is illustrated in Equations (1-4). Thus, the internal blind vias introduced inside the filter separate the electro-magnetic field, thereby slowing the wave, while increasing the permittivity and also permeability, as a result reducing the longitudinal and lateral dimensions. In fact the center frequency of SIW filter decreased from $39.5 \mathrm{GHz}$ to $33.5 \mathrm{GHz}$, thereby shifting the center frequency by $6 \mathrm{GHz}$.

$$
\begin{aligned}
& L^{y}=L_{0}^{y}+\Delta L \\
& C^{y}=C_{0}^{y}+\Delta C \\
& \mu_{\text {eff }}=\frac{L^{y}}{\mu_{0}} \\
& \varepsilon_{\text {eff }}=\frac{C^{y}}{\varepsilon_{0}}
\end{aligned}
$$

where, $L_{0}^{s}$ represents the inductance of the basic filter without slow wave.

$L^{J}$ represents the lumped inductance of the filter after incorporating slow wave.

$C_{0}^{l}$ represents the capacitance of the basic filter without slow wave.

$C$, represents the capacitance of the filter with slow wave.

This shows that a filter of smaller size, which was originally designed for higher frequency band (here $39.5 \mathrm{GHz}$ ) is now being used in a slightly lower frequency (here $33.5 \mathrm{GHz}$ ) with the same small size. This is apparent from Figure 9 which shows a comparison of the filter performance with and without the slow wave. It could be observed from the comparison that the insertion loss is

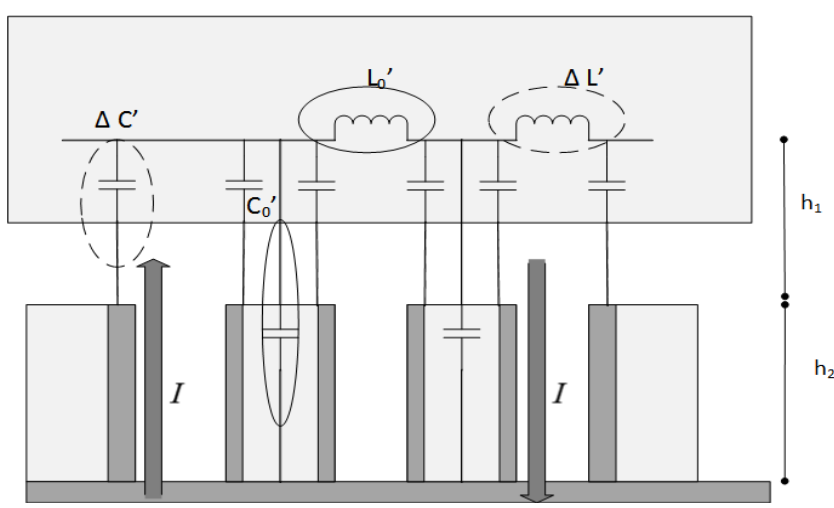

(a)

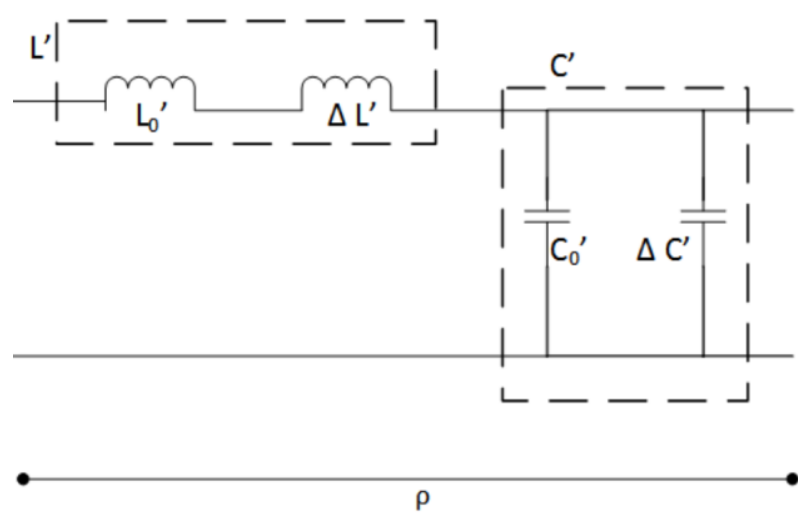

(b)

Figure 8: (a) Lumped circuit with effective circuit elements. (b) Complete lumped element equivalence for a unit cell

lower by $2 \mathrm{~dB}$ and bandwidth is wider by $0.5 \mathrm{GHz}$. Quality factor and group delay are important parameters in filters. Quality factor of the SW SIW band pass filter is 506. A comparison of group delay of filters is shown in the Figure 10. The value of group delay in the slow wave filter is less compared to the value in the filter without slow wave. This shows that the wave is longer time to travel from one port to the other port in the slow wave filter. The delay is added due to the physical separation of the electric and magnetic fields in the structure. Thus the slow wave concept is also validated from the values of group delay in both filters.

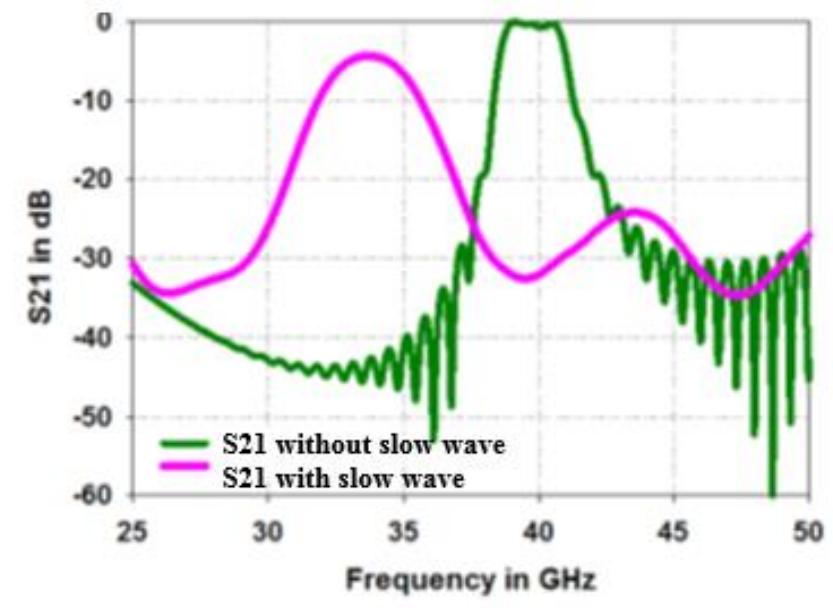

Figure 9: Comparison of transmission characteristics 


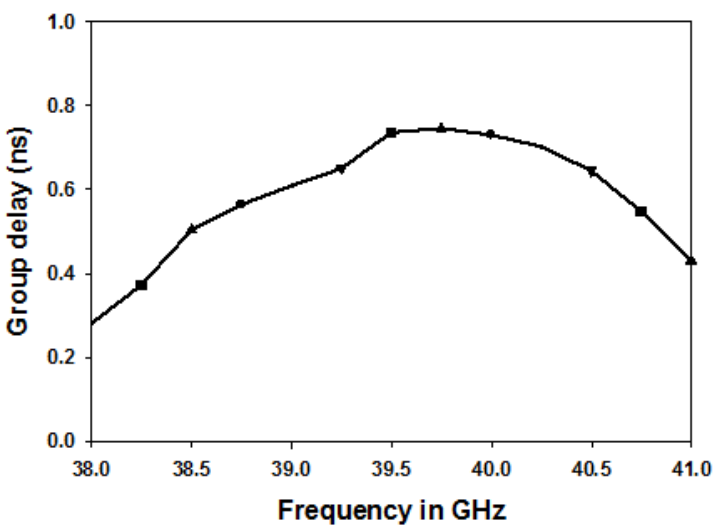

(a)

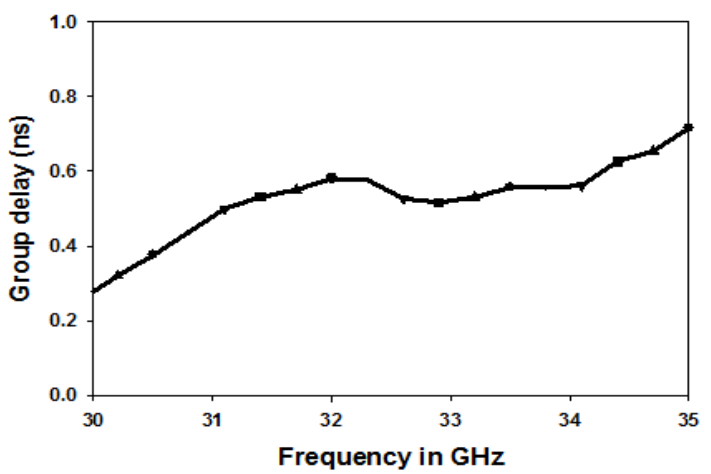

(b)

Figure 10: (a) Group delay in SIW band-pass filter (b) Group delay in SW SIW band-pass filter.

\section{Conclusion}

A miniaturized band pass inductive post filter in SIW technology is presented in this paper. The miniaturization was achieved using slow wave phenomenon. The slow wave technique was investigated with a double layer topology, wherein several rows of internal metallized vias are introduced in the bottom layer connected to the bottom conducting plane. The number of rows of internal metallized vias was chosen with a parametric study. The concept of slow wave, not used before for miniaturization of inductive post band pass filter, shows that it is possible to achieve smaller longitudinal dimensions resulting in an area reduction , by almost maintaining the same filter characteristics. Analysis showed that the SIW filter is $21.6 \%$ and $34.6 \%$ miniaturized in size and area respectively. The response of the filter spans the Ka band, hence, the filter can be used for satellite communication application. A quality factor of 506 is achieved for the miniaturized filter.

\section{References}

[1] Qiao-Li Zhang, Wen-Yan Yin, Sailing He and Lin-Sheng Wu, Com pact Substrate Integrated Waveguide (SIW) Bandpass Filter With Complementary Split-Ring Resonators (CSRRs), IEEE Microwave and Wireless Components Letters, Vol. 20, No. 8, August 2010.

[2] Weiping Li, Zongxi Tang, and Xin Cao,Design of a SIW Bandpass Filter Using Defected Ground Structure with CSRRs, Hindawi Publishing Corporation, Active and Passive Electronic Components Volume 2017.

[3] X.-C. Zhang, Z.-Y. Yu and J. Xu,Novel Band-Pass Substrate Integrated

Waveguide (SIW) Filter Based On Complementary Split Ring Resonators (CSRRs),Progress In Electromagnetics Research, PIER 72, 3946, 2007.

[4] Yongmao Huang, Zhenhai Shao and Lianfu Liu,A Substrate Integrated Waveguide Bandpass Filter Using Novel Defected Ground Structure Shape,Progress In Electromagnetics Research, Vol. 135, $201-213,2013$
[5] Y.M. Huang, Z.S. He, P.K. Li, Z.H. Shao, C.J. You, and D. Jiang,A Bandpass Filter Using HMSIW-DGS Cell,PIERS Proceedings, Guangzhou, China, August 25-28, 2014.

[6] Yong Mao Huang, Zhenhai Shao, Zhaosheng He, Chang Jiang You, and Di Jiang,A Bandpass Filter Based on Half Mode Substrate Integrated Waveguide-to-Defected Ground Structure Cells, Hindawi Publishing Corporation, International Journal of Antennas and Propagation, Volume 2015.

[7] Zhibing Liang, Kuandong Gao, Yongmao Huang, Lianfu Liu, Zhen. Hai. Shao, A Ka Band Broadband Bandpass Substrate Integrated Waveguide Filter,4th International High Speed Intelligent Communication Forum (HSIC), 2012.

[8] Sourav Moitra, Basudeb Mondal, Jhuma Kundu, Asish Kumar Mukhopadhyay and Anu Kumar Bhattacharjee,Substrate Integrated Waveguide (Siw) Filter Using Stepped-Inductive Posts For KuBand Applications,Institution of Engineering and Technology, 2013.

[9] Cheng Zhao, Christophe Fumeaux, Thomas Kaufmann, Yingbo Zhu, Ali K. Horestani, and Cheng-Chew Lim,A General Design Method for Band-pass Post Filters in Rectangular Waveguide and Substrate Integrated Waveguide,International Symposium on Antennas and Propagation, 2015.

[10] Qing-Tao Huang, Qing-Xin Chu, Kai Wang, Sai Waiong,,Multimode

Band-pass Filter with Four Transmission Zeros on SIW Structure,IEEE International Conference on Computational Electromagnetics (ICCEM), 2016

[11] Mohamad Khalil, Mahmoud Kamarei,Jalal Jomaah and Hussam Ayad,Compact Multi-layer Band-Pass Filter in Substrate Integrated Waveguide (SIW) Technology, IEEE Middle East Conference on Antennas and Propagation (MECAP), September 2016

[12] Cao Tri Bui, Petr Lorenz, Mustafa Saglam, Wilhelm Kraemer and Rolf H. Jansen,Investigation of Symmetry Influence in Substrate Integrated Waveguide (SIW) Band-Pass Filters using Symmetric Inductive Posts,Proceedings of the 38th European Microwave Conference, 2008.

[13] Ahmed Rhbanou and Seddik Bri,Design of Substrate Integrated Waveguide Pass Filter at [33-75] GHz Band,International Journal of Engineering and Technology (IJET), Vol.6, No.6, Dec 2014-Jan 2015.

[14] Feng Xu and Ke Wu,Guided-Wave and Leakage Characteristics of Substrate Integrated Waveguide,IEEE Transactions on microwave theory and techniques, Vol.53, No.1, January 2005.

[15] Alejandro Niembro-Martin, Victoria Nasserddine, Emmanuel Pistono, Hamza Issa, Anne-Laure Franc, Tan-Phu Vuong, and Philippe Ferrari,Slow-Wave Substrate Integrated Waveguide,IEEE Transactions on microwave theory and techniques, Vol.62, No.8, August 2014.

[16] Matthieu Bertrand, Zhanhao Liu, Emmanuel Pistono, Darine Kad dour, Philippe Ferrari,A Compact Slow-Wave Substrate Integrated Waveguide Cavity Filter,Microwave Symposium (IMS), 2015 IEEE MTT-S International, 17-22 May 2015.

[17] Hemendra Kumar, Ruchira Jadhav, and Sulabha Ranade,A Review on Substrate Integrated Waveguide and its Microstrip Interconnect,IOSR Journal of Electronics and Communication Engineering (IOSR-JECE), Volume 3, Issue 5, Sep. Oct., 2012.

[18] Woon-Gi Yeo, Tae-Yoon Seo, Jae W. Lee, and Choon Sikho,HPlane

Sectoral Filtering Horn Antenna PCB Substrates Using Via Fences at Millimeter-Wave,Proceedings of the 37th European Microwave Conference, October 2007.

[19] Ahmed Rhbanou, Mohamed Sabbane,Design of K-Band Substrate Integrated Waveguide Band-Pass Filter with High Rejection,Journal of Microwaves, Optoelectronics and Electromagnetic Applications, Vol. 14, No. 2, December 2015.

[20] Martin Coulombe, Hoang V. Nguyen and Christophe Caloz,Substrate

Integrated Artificial Dielectric (SIAD) Structure for Miniaturized Mi crostrip Circuits,IEEE Antennas and Wireless Propagation Letters, Vol.6, 2007.

[21] N.Marcuvitz,Waveguide Handbook,New York Dover Publications, Inc.

[22] Bong S. Kim, Jae W. Lee, Kwang S. Kim, Myung S. Song,PCB Substrate Integrated Waveguide-Filter Using Via Fences at Millimeterwave, IEEE MTT-S, 2004.

[23] Hun Nam, Hyeonjin Lee, and Yeongseog Lim,A Design and Fabrication of Bandpass Filter using Miniaturized Microstrip Square SIR , Pro ceedings of IEEE Region 10 International Conference on Electrical and Electronic Technology, 2001. 